MPI-PhT/94-75

DAMTP R94/43

gr-qc/9411008

November 1994

\title{
A Parallelizable Implicit Evolution Scheme for Regge Calculus
}

\author{
John W. Barrett ${ }^{1}$, Mark Galassi ${ }^{2}$, Warner A. Miller ${ }^{3}$, \\ Rafael D. Sorkin ${ }^{4}$, Philip A. Tuckey ${ }^{5}$ and Ruth M. Williams ${ }^{6}$
}

\begin{abstract}
The role of Regge calculus as a tool for numerical relativity is discussed, and a parallelizable implicit evolution scheme described. Because of the structure of the Regge equations, it is possible to advance the vertices of a triangulated spacelike hypersurface in isolation, solving at each vertex a purely local system of implicit equations for the new edge-lengths involved. (In particular, equations of global "elliptic-type" do not arise.) Consequently, there exists a parallel evolution scheme which divides the vertices into families of non-adjacent elements and advances all the vertices of a family simultaneously. The relation between the structure of the equations of motion and the Bianchi identities is also considered. The method is illustrated by a preliminary application to a 600-cell Friedmann cosmology. The parallelizable evolution algorithm described in this paper should enable Regge calculus to be a viable discretization technique in numerical relativity.
\end{abstract}

\footnotetext{
1 Mathematics Department, The University of Nottingham, University Park, Nottingham NG7 2RD, UK

2 Space Data Systems Group, Los Alamos National Laboratory, Los Alamos, NM 87545, USA

3 Theoretical Division, Los Alamos National Laboratory, Los Alamos, NM 87545, USA

4 Physics Department, Syracuse University, Syracuse, NY 13244-1130, USA

5 Max-Planck-Institut für Physik, Föhringer Ring 6, D-80805 München, Germany

${ }^{6}$ DAMTP, Silver Street, Cambridge CB3 9EW, UK
} 


\section{Numerical relativity via (fully 4-dimensional) Regge calculus.}

Much current activity in numerical relativity is centered around making predictions which can be tested by the proposed Laser Interferometry Gravitational Observatory (LIGO). ${ }^{1,2}$ There is a need to solve Einstein's equations numerically for many physical situations which could give rise to gravitational waves, so that data from LIGO can be interpreted, and used, if appropriate, as evidence for the existence of black holes. More generally, numerical solutions of Einstein's equations are invaluable for the understanding of astrophysical data, and for guidance as to what experiments to undertake.

Methods of solving Einstein's equations numerically include finite difference schemes and finite element schemes. Regge calculus is a type of finite element method, and in this paper we shall describe a way of casting it into the form of a highly efficient tool of numerical relativity.

The basic idea of Regge calculus is the division of spacetime into simplicial cells with flat interior geometry. ${ }^{3}$ The dynamical variables are the edge-lengths of the simplices, and the curvature, which is restricted to the "hinge simplices" of codimension two, can be expressed in terms of the defect angles at these hinges, where the flat cells meet. Variation of the action leads to the simplicial form of Einstein's equations. The convergence of the Regge action and equations to the corresponding continuum quantities has been investigated thoroughly and has been shown to be satisfactory under quite general conditions. ${ }^{4-9,22,30}$ Regge calculus has been applied to a large variety of problems in classical and quantum gravity (see Ref. 10 for a review). Numerical applications in 3+1 dimensions have been mainly to problems with symmetry and no general code has been developed. ${ }^{11-15,37}$ This is also the case in the alternative approach known as null-strut calculus, which builds a spacelike-foliated spacetime with the maximal number of null edges. ${ }^{16-20}$ Although null-strut calculus was used first to demonstrate numerically the approximate diffeomorphism freedom in Regge calculus, ${ }^{18}$ and, except for the work described in ref. 30, was the first fully $3+1$ dimensional numerical scheme implemented ${ }^{21}$, we have not found a way to adapt it to the evolution scheme described in this paper. Therefore, unless we find an alternative decoupling scheme the standard approach to Regge calculus appears to be much more tractable numerically.

In this paper, we describe how the implementation of ideas developed almost twenty years ago $^{22}$ results in a much more efficient way of using Regge calculus in numerical relativity. The evolution of a spacelike hypersurface can be achieved one vertex at a time, or in parallel for vertices which are not connected by an edge. This scheme is described in Sec. 2, and the role of the Bianchi identities and the resulting freedom to specify lapse and shift information are discussed in Sec. 3. In Sec. 4, it is shown how the scheme works for one of the lattices originally suggested by Sorkin, and in Sec. 5 it is shown how it is related to previous evolution schemes using Regge calculus. Sec. 6 consists of a numerical example, and Sec. 7 contains some concluding remarks.

\section{General description of the evolution scheme.}

In a sense, the scheme which we will describe now is not a new way of doing Regge calculus, but rather a new understanding of the elegant way in which the standard evolution 
scheme works in Regge calculus. It is based on what is described by Sorkin for two particular lattices; ${ }^{22}$ the general validity of these ideas was realized only recently and is summarized by Tuckey. ${ }^{23}$

Consider a time evolution problem in Regge calculus, and suppose that all the edgelengths of a solution, up to and including a given triangulated spacelike hypersurface are known. Then, with an appropriate continuation of the triangulation into the future, any vertex on that surface can be evolved forward to the corresponding vertex on the next hypersurface by solving only a small set of equations from the immediate neighborhood of the vertex.

To see how this works, look first at what happens in $2+1$ dimensions. Consider part of the two dimensional spacelike surface surrounding one vertex (the star of that vertex) (Fig. 1). To advance this vertex to the next hypersurface, we introduce a new vertex "above" it; we connect this to the chosen vertex by a "vertical edge," and by "diagonal" edges to all the vertices on the original surface to which the chosen vertex was connected. The result of this is to stick a tetrahedron, with apex at the new vertex, on each of the triangles surrounding the original vertex (Fig. 2). It is rather like erecting a tent above the chosen vertex, with the vertical edge as the tent pole and the star (within the spacelike hypersurface) of the chosen vertex as the floor of the tent.

In order to evolve the new vertex, we need to find the lengths of the new edges. Recall that the empty space Regge equation for an edge $L_{i}$,

$$
\sum_{h} \frac{\partial A_{h}}{\partial L_{i}} \epsilon_{h}=0,
$$

where the sum is over hinges $h$, with $A_{h}$ the volume content of the simplicial hinge, and $\epsilon_{h}$ the defect angle there, involves only the edge lengths of the simplices containing $L_{i}$. Thus the only equations which involve the new edges and edges already known are those for the new vertical edge and for the edges in the original surface which radiate from the chosen vertex. Since by construction there is one such edge corresponding to each new diagonal edge, there are precisely the same number of equations as unknown edge lengths. Thus one might think that we could solve directly for the edges. However, as we shall explain, the equations are expected to have an approximate functional dependence among them, which means that (for sufficiently fine triangulations) it will probably be better to use the effective lack of determination to choose lapse and shift, as in the continuum.

The construction just described can be generalized immediately to $3+1$ dimensions. We choose a vertex on the given spacelike hypersurface, introduce a new vertex above it and connect the new vertex by a "vertical" edge to the chosen vertex and by "diagonal" edges to all the vertices in the original hypersurface to which the chosen vertex was joined. Each tetrahedron in the original surface which contains the chosen vertex now has based on it a 4-simplex, with apex at the new vertex. Note that again in this tent-like construction (Fig. 3), there is one diagonal corresponding to each edge in the original surface radiating from the chosen vertex. We now use the variational equations for these edges in the original surface and for the vertical edge; the only unknown edges which these equations involve are the new vertical edge and the diagonal edges and there is the same number of equations as 
unknowns, so in principle we could again solve uniquely for the unknown edges. However in practice we shall again ignore some of the equations and instead specify conditions on the lapse and shift. (Although fundamentally all the variational equations have the same status, they play different roles in the chosen advancement scheme. Those associated with the "vertical" edges involve only the region between successive spacelike hypersurfaces and are in that sense constraint-type equations, whereas those associated with the other edges stretch between three such hypersurfaces and are in that sense evolution-type equations. Nevertheless, eliminating 3 degrees of freedom in favor of freely specifiable shift information effectively treats 3 combinations of the latter equations as "constraint-like" as well.)

So far, we have described how to advance just one vertex in time. The method can clearly be used to evolve the entire hypersurface, by advancing the vertices one-by-one (see Fig. 4 for a representation of this process in $1+1$ dimensions). This process is completely general; it can be used for any triangulation of a hypersurface having any topology.

Advancing the vertices one-by-one will not ordinarily be the most efficient way of evolving a hypersurface. If any two vertices in a hypersurface are not connected by an edge, then they can be evolved to the next surface at the same time without interfering with each other, as shown schematically in Fig. 5. Thus the method is obviously parallelizable.

Whichever the order in which the vertices are advanced, each vertex has a unique predecessor and successor, and so the structure always contains lines through it connecting the vertices in this way. Thus it is "washing line topology." The particular order of advancement chosen hangs the washing on the line in a particular way.

After a vertex has been evolved one step, the new exposed 3-surface, which is made up of most of the old hypersurface plus the exposed tetrahedral faces of the 4-simplices which were added, has the same triangulation as the original hypersurface. This is because the way that the 4-simplices are constructed ensures that each tetrahedron in the original surface has a corresponding tetrahedron in the new surface. Thus the structure of the exposed 3-surface is the same at all stages of the time evolution calculation, even though the structure of the 4-dimensional slice beneath that 3-surface depends on the order in which the vertices were advanced to it. This beautifully simple structure could however be seen as a limitation if one wants to allow less "orderly" geometries where the triangulation or even the topology changes in time. One might wish to generalize the scheme to something more flexible where two washing lines knot together or one branches out into two. It is possible that this could be achieved by using a sequence of elementary moves on the triangulation. ${ }^{24}$

In a time evolution calculation the "vertical" edges always go between hypersurfaces. However the "diagonal" edges initially lie in a new hypersurface but later may be regarded as going between surfaces when more of the original surface is evolved (See Fig. 5). What restriction does causality place on them? Consider the "tent" above a chosen vertex; we are trying to calculate the 4-geometry within it from data given on (and below) the base of the tent, so the tent as a whole ought to lie within the domain of dependence of its base. Even though the Regge equations are in one sense being solved "implicitly" we expect that for the solution to be stable, this causality condition should be satisfied, i.e. the whole of the tent should be contained within the "light pyramid" on its spacelike base (Fig. 6). In particular, the diagonal edges, which form the boundary of the tent, must be spacelike. 
On the other hand, the vertical edge could in principle be timelike, null or spacelike. Since it seems that the diagonal edges will have to be spacelike, we see that all the 3 -surfaces, even the ones which could be thought of as intermediate stages in the evolution of some starting hypersurface to the next, will actually be spacelike. A picture emerges of a whole pile of "concertina'd" spacelike 3-surfaces lying on top of each other in a lot of places, with occasional gaps. To use yet another metaphor, it looks like puff pastry.

The requirement that the diagonal edges be spacelike indicates that it will not be a good idea to advance one particular vertex by several steps while not evolving the surrounding ones. In that case, the diagonal edges would be likely to become timelike rather soon, and long narrow triangles would be produced, which have proven not to be good in numerical calculations, ${ }^{17}$ and are also known to be bad for convergence. ${ }^{38}$

Finally let us mention a possible application of these same ideas to the initial value problem. Since in the formulation just described there is no meaningful distinction between initial value and time evolution equations, one could contemplate building up an initial set of edge-lengths by the same process of "advancing vertices" described above in the context of time evolution. The only difference would be that certain edge-lengths which occurred in the equations would now be unknown, and therefore freely specifiable as initial data, rather than given by the results of prior evolution. This would seem to lead to a parallelizable method for solving the initial value equations as well. On the other hand, one would not expect this to be possible, since the initial value problem has a fundamentally elliptic character in contrast to the time evolution problem, which in the continuum is hyperbolic, and therefore effectively local. Perhaps the resolution is that an initial value solution produced by any such scheme of "advancing" individual vertices would behave unstably, but at any rate it seems a question worthy of further study.

\section{Bianchi Identities and Lapse and Shift.}

Let us look now at the counting involved in the type of evolution scheme just described. At first sight there appears to be an exact match at each vertex between the number of unknown variables (edge-lengths) and the number of equations available to determine these. Two obvious and related questions arise. Firstly, are all the equations independent? Secondly, what has become of the freedom to choose lapse functions and shift vectors which exists in the continuum theory?

This matching of the numbers of unknowns and equations precisely mirrors the continuum theory where we have ten unknown metric components $\left(g_{\mu \nu}\right)$ and ten Einstein equations $\left(G_{\mu \nu}=8 \pi T_{\mu \nu}\right)$ available to determine them. However, we know that in the continuum not all of the equations are independent. There is a four-fold redundancy corresponding to the contracted Bianchi identities $(\nabla \cdot G=0)$. Thus in the continuum theory we are free to impose four conditions per point on the metric (one lapse condition and three shift conditions) when evolving from one 3-geometry to the next. Furthermore, the constraint equations once satisfied on the initial 3-geometry will automatically remain solved in the evolution. This is analogous to conservation of energy and momentum. Therefore in practice we must solve six evolution equations for the six metric parameters that remain.

These continuum relationships have their counterparts in Regge calculus and in particular when applied to the scheme described in the previous section. In Regge calculus, 
the ordinary Bianchi identities correspond (in four dimensions) to the statement that the product of the rotation matrices for the hinges meeting on any edge is the identity transformation. ${ }^{3}$ In the limit of small defect angles, it can be shown that this is equivalent to the usual continuum Bianchi identities. In this case the equivalent of the contracted Bianchi identity is that the sum over all edges meeting at a vertex, of the equivalent of the Einstein tensor along that edge, is zero. ${ }^{7,25}$ However, this vector equation is an approximate identity; it is only exact in the linearized case or in the continuum limit. Thus it provides four approximate equations per point, relating the Regge equations for the edges at that vertex. This is associated with an approximate symmetry of the theory.

The implications of the contracted Bianchi identities for our evolution scheme therefore go as follows. When we evolve a vertex we will have $N$ equations for $N$ unknown edgelengths. Because of the approximate four-fold redundancy per vertex we expect to be able to use $N-4$ of the equations together with four externally imposed lapse and shift conditions to solve for the unknown edges. For example, we might simply choose the vertical edge-length as the lapse degree of freedom, and three of the diagonal edges as the shift, and ignore the corresponding Regge equations associated with the vertical edge and the corresponding edges in the tent "floor".

In a study of the Kasner cosmology, we have in fact imposed more sophisticated lapse and shift conditions that involve four algebraic relations among the $N$ variables corresponding to the standard definitions of lapse and shift. ${ }^{26,29}$ We have observed numerically that the conservation of energy-momentum (in the sense of automatic satisfaction of the unimposed equations) improves when we refine the lattice, and that the Jacobian of second partial derivatives of the action becomes more and more singular as we approach the continuum limit, in agreement with earlier theoretical predictions. ${ }^{7,22,25,29,30,35,39,40}$

The approach developed here for evolution is in may ways similar to the finite difference algorithm used by Kurki-Suonio, Laguna and Matzner ${ }^{27}$ however, our discretized equations are written in an implicit form. To our knowledge this is the first implicit numerical scheme in $(3+1)$-dimensional numerical relativity.

\section{Sorkin's Lattice.}

We now illustrate the scheme by looking at one of the lattices for which it was originally suggested. ${ }^{22}$ Consider first the 2-dimensional version, for a triangulation of $S^{1} \times R$. We label vertices by $[t]$ or $\left[t^{*}\right]$ with $t \in Z$, and join them according to the following rules: $\left[t_{1}\right]$, $\left[t_{2}\right]$ or $\left[t_{1}^{*}\right],\left[t_{2}^{*}\right]$ are joined if $0<\left|t_{1}-t_{2}\right| \leq 2$; and $\left[t_{1}\right],\left[t_{2}^{*}\right]$ are joined if $0<\left|t_{1}-t_{2}\right| \leq 1$. The resulting lattice is shown in Fig. 7 . The triangles are of the form $[t, t+1, t+2]$ where $t$ and $t+2$ were both starred or unstarred, and $t+1$ may or may not be starred. Clearly all vertices are equivalent. What is happening here is that $S^{1}$ is being represented by a square, and pairs of opposite vertices (e.g. $[0]\left[0^{*}\right]$ and $\left.[1]\left[1^{*}\right]\right)$ are staggered in time.

In four dimensions, we consider a triangulation of $S^{3} \times R$ in which $S^{3}$ is tessellated by $\beta_{4}$, which has 8 vertices and 16 tetrahedra. (More generally, one can use the family of posets depicted in figure 5 of reference 36 to produce analogous triangulations for all the $n$-spheres $S^{n}$, each such triangulation being the so-called "order-complex" of the corresponding poset. The square is the $n=1$ reprsentative of this family and $\beta_{4}$ is the $n=3$ representative.) The vertices are labeled as in the 2-dimensional case and the rules for joining them are: 
$\left[t_{1}\right],\left[t_{2}\right]$ or $\left[t_{1}^{*}\right],\left[t_{2}^{*}\right]$ are joined if $0<\left|t_{1}-t_{2}\right| \leq 4$; and $\left[t_{1}\right],\left[t_{2}^{*}\right]$ are joined if $0<\left|t_{1}-t_{2}\right| \leq 3$. Again all vertices are equivalent, and we may regard them as a succession of pairs $[0]\left[0^{*}\right]$, $[1]\left[1^{*}\right],[2]\left[2^{*}\right], \ldots$. These pairs are the antipodal vertices of $\beta_{4}$ which in some sense are staggered in time. The 4-simplices are of the form $[t, t+1, t+2, t+3, t+4]$ where either $t$ and $t+4$ are both starred or both unstarred. Each intermediate vertex may be starred or unstarred.

The time evolution for this lattice proceeds as follows. We suppose that all lengths up to and including vertices [3] and [3*] are known. Now consider vertex [4], which is the vertex "above" [0]. [4] is joined to seven of the "earlier" vertices by the edges going "backwards" from it,

$$
[04][14]\left[1^{*} 4\right][24]\left[2^{*} 4\right][34]\left[3^{*} 4\right] .
$$

These are the new edges in the tent above vertex [0], the first of them being the vertical edge and the others being the diagonal edges of this tent. Then consider the "forward"-going edges from vertex [0]: namely

$$
[01]\left[01^{*}\right][02]\left[02^{*}\right][03]\left[03^{*}\right][04]
$$

of which the first six are the horizontal edges of the tent and the last is the vertical edge. We can check that the Regge equations for these edges involve only edges known already, plus the new edges listed above. Thus we have seven equations for seven unknowns, and if wanted to treat the equations as truly independent, we could solve uniquely for the new edge-lengths. By doing so we would be letting the equations "choose their own gauge," and it is conceivable that they would make a reasonable choice, since we are rather far from the continuum limit. If, on the contrary, the approximate symmetries in Regge calculus manifested themselves in an unstable behavior of the resulting solutions, then we could instead eliminate 4 of the seven equations in favor of freely specifiable lapse and shift data, as discussed above. With either procedure we would, in the language of Sec. 2, have chosen vertex [0] and advanced it to vertex [4].

Notice that the procedure for advancing to vertex [4] involves neither vertices $\left[0^{*}\right]$ or $\left[4^{*}\right]$ so in fact we could carry through the advance of vertex $\left[0^{*}\right]$ to vertex $\left[4^{*}\right]$ at exactly the same time. This is because the vertices $[t]$ and $\left[t^{*}\right]$ have no edge in common.

We can now repeat the process to find everything up to and including vertices [5] and $\left[5^{*}\right]$ and so on. At each step we will have two sets of 7 equations in 7 unknowns to solve (each set containing three "dynamical relations" and four "gauge conditions").

Before performing the time evolution, we must solve the initial value problem. The equations used in advancing to vertices [4] and [ $\left.4^{*}\right]$ involve edges going back as far as $[-3]$ and $\left[-3^{*}\right]$, so for initial data, we need to know the lengths of all the edges between $[-3]$, $\left[-3^{*}\right]$ and $[3],\left[3^{*}\right]$. This involves a total of 66 edges. Amongst these are just 18 edges whose Regge equations involve only edges from the 66 . Thus one way of proceeding is to specify freely 48 edges and then solve the 18 equations for the remaining ones.

\section{Comparison with other numerical work in $3+1$ Regge calculus.}

Before attempting to relate other approaches to that of this paper, let us emphasize the differences. Typical of earlier $3+1$ approaches are those of Collins and Williams, ${ }^{11}$ 
Porter ${ }^{13,14}$ and Brewin. ${ }^{33}$ The basic idea is to take successive spacelike hypersurfaces triangulated by tetrahedra, and to join corresponding vertices by timelike lines, thus constructing a set of 4-prisms which tessellate the spacetime. The shape of a 4 -prism is not uniquely determined by its edge lengths, so it is necessary to give further information to eliminate the floppiness. In the study of the Friedmann universe by Collins and Williams, ${ }^{11}$ there is sufficient symmetry to determine the shapes of the prisms. In the work of Porter ${ }^{13,14}$ and later of Dubal, ${ }^{28}$ angles in the faces of the prisms are introduced as extra variables related to the extrinsic curvature. In all of these approaches, the Regge equations fall into two categories, evolution equations which arise from variation of spacelike edges, and constraint equations which come from variation of timelike edges. The equations are coupled together in such a manner as to make local solution impossible.

Apart from the different triangulations and the appearance of distinct categories of equations, these methods also differ from the Sorkin approach in that the spacelike hypersurfaces of a distinguished family are clearly displayed at different times, whereas those in the Sorkin method described in Sec. 2 are represented as staggered in time, in the manner of puff-pastry. In the latter method, a subset of the surfaces may be identified with those of the former method (though in general the choice of subset is not unique).

We now describe how the prism construction can be modified so that the general method of Sec. 2 is applicable to it and then describe the relationship in detail for the particular lattice of Section 4 above.

The modification required is a very simple one; all that is required is the addition of enough diagonals or braces to divide each prism into four 4-simplices. (We note with apology the possibility for confusion between these "diagonals" and the "diagonal edges" discussed up until now. The "diagonals" here are defined with respect to a particular family of non-intersecting 3-dimensional hypersurfaces. The "diagonal edges" discussed previously are defined only with respect to a particular evolution step.) There are various prescriptions for doing this. One possibility is to order all the vertices in the upper hypersurface (which forms the top layer of all the prisms), and then connect the first vertex in each upper simplex (for all simplices of all dimensions) to every vertex of the corresponding simplex in the lower hypersurface. Then the $k$-simplices are just the subsets of a single cell spanned by vertices all of which are connected together. The resulting simplicial complex is then exactly what would be obtained by performing the evolution process of Sec. 2 on the lower hypersurface, advancing the vertices in exactly the order imposed on the upper hypersurface. (A similar construction is possible in any dimension, see for example ref. 41 and section 3 of ref. 36.)

Note that what we are claiming here is not that the prism methods are equivalent to that of Sec. 2, but rather that the lattices used can be modified to fit the new method. The prism methods per se are quite distinct dynamically, involving for example the use of angles as variables. Once the diagonal edges have been introduced, there is no point in using any extra variables, since one returns to the situation in conventional Regge calculus where the edge lengths completely specify the geometry. Clearly, the curvature in the 4-prism based geometries is less general than that allowed after the subdivision into 4-simplices, since in the prism approach the curvature is constant across a 2-prism, which is not true when it is split into 2 triangles. 
Let us now show how to introduce the diagonals into the prism construction for a particular lattice, which reproduces the lattice described in Sec. 4 . Consider $\beta_{4}$, the 16 -cell tessellation of $S^{3}$. Label the vertices at one moment of time [0], [1], [2], [3], [0*], [1*], [2*], [3*] (to tie in with Sorkin's notation), as shown in Fig. 8. At the next moment of "time", label the vertices $[4],[5],[6],[7],\left[4^{*}\right],\left[5^{*}\right],\left[6^{*}\right],\left[7^{*}\right]$. We now join corresponding vertices (those differing by 4 ) at successive times to obtain a collection of 4 -prisms which we now divide into 4-simplices in the following way. In each quadrilateral face within a prism, we draw in a diagonal joining the two vertices with nearer numbers. For example in the prism with lower tetrahedral face $\left[0^{*} 1^{*} 23\right]$ and upper tetrahedral face $\left[4^{*} 5^{*} 67\right]$ (Fig. 9), we insert the diagonals $\left[1^{*} 4^{*}\right],\left[24^{*}\right],\left[25^{*}\right],\left[34^{*}\right],\left[35^{*}\right]$ and [36]. The prism is now divided into $4-$ simplices $\left[0^{*} 1^{*} 234^{*}\right],\left[1^{*} 234^{*} 5^{*}\right],\left[234^{*} 5^{*} 6\right]$ and $\left[34^{*} 5^{*} 67\right]$. It is clear that the rule by which vertices are joined produces exactly the same lattice as in the Sorkin case. This means that when we apply the method of Sec. 2, the dynamics of the evolution procedure are exactly the same; to evolve to the next hypersurface we have to solve two sets of seven equations for seven unknowns, four times over. The interpretation of the procedure may be slightly different here, as already pointed out, in that there is no suggestion of the designated spacelike hypersurfaces being staggered in time or concertina'd in shape.

The initial value problem here differs slightly from the Sorkin case. In order to evolve the spacetime, we need to specify two spacelike slices and the spacetime between them. This involves 24 spacelike edges in each hypersurface, plus 8 timelike edges and 24 diagonals in between, making a total of 80 edges. The equations involving only these edges are those for the timelike edges and diagonals, a total of 32 equations. Thus we may specify freely 48 edges (in exact agreement with the Sorkin case) and use the available equations to solve for the other 32. The difference arises because the initial value problem identified here corresponds to the minimal initial value problem identified in Sec. 2, together with the addition of the two evolution steps of vertex [3] to [7] and $\left[3^{*}\right]$ to $\left[7^{*}\right]$, which provides the additional 14 equations and 14 unknowns.

One of the main points of this section was to show that local evolution (and sometimes parallel evolution) is possible, and indeed natural, in the previous work on $3+1$ Regge calculus using prisms, provided the prisms are divided into 4-simplices by the insertion of extra edges. The possibility of advancing several vertices at the same time depends on the symmetry of the lattice. We have already seen how this happens for the lattice based on $\beta_{4}$, and we shall end this section by looking briefly at how it works for the other regular tessellations of $S^{3}$.

For $\alpha_{4}$, the 5 -cell triangulation of $S^{3}$, there are 5 vertices and 10 edges. In this case, since all the vertices are connected to each other, it is not possible to advance any pair simultaneously. The evolution procedure can be shown to involve solving 5 equations for 5 unknowns, 5 times over, to advance to the next spacelike hypersurface.

The 600-cell triangulation of $S^{3}$ can be built up from 30 blocks of 20 tetrahedra meeting at single vertex. Clearly these blocks meet the condition that none of the central vertices shares an edge, and so we can advance the 30 central vertices at the same time. We then consider another division into 30 blocks, and advance their central vertices simultaneously. Since the total number of vertices is 120 , the process will have to be repeated 4 times to evolve a spacelike hypersurface to the next distinct spacelike hypersurface. Now 
in each hypersurface, 12 edges meet at each vertex; we use the Regge equations for those edges and for the vertical edge from the vertex to its advancement in the next hypersurface, to solve for that vertical edge and the 12 diagonal edges involved. Thus the whole evolution process from one surface to the next distinct surface involves solving four times 30 sets of 13 equations for 13 unknowns, making a total of 1560 edges to be solved for ultimately. These are made up of 720 edges in the new spacelike hypersurface, 120 vertical edges and 720 diagonals (which are also spacelike edges, playing the roles first of diagonal edges and then horizontal edges in the evolution steps).

It is interesting to note the relationship between the number of variables to be solved for at each stage, and the number of cells in the triangulation. The cases mentioned show the following progression:

tetrahedra: $5,16,600, \infty$; $\quad$ unknowns: $5,7,13,15$.

Here the last entry is taken from Ref. 22 for the $R^{4}$ triangulation and is the same number obtained in the asymptotic limit for the "quantity-production" lattice introduced in Ref. 17. (However, this by no means proves that the average number of equations per vertex is necessarily 15 in the limit of an arbitrarily large number of tetrahedra. For example, the average number of edges per vertex for an infinitely barycentrically-subdivided triangulated 3 -dimensional hypersurface is $13 / 2$, which leads in our evolution scheme to $13+1=14$ equations per vertex, rather than 15; and doing the subdivision directly on the 4-dimensional complex for comparison, would lead to the still smaller value of $25 / 2$ $=12.5$ edges or Regge equations per vertex. On the other hand, neither of these subdivision schemes is physically appropriate since repeated barycentric subdivision produces infinitely squashed simplices in the limit of infinite refinement.)

\section{Preliminary Numerical Example.}

We now illustrate the scheme described in this paper by applying it to the evolution of the 600-cell tessellation of $S^{3}$ mentioned in the previous section. Matter will be included in the form of pressure-less dust, and we will look for homogeneous solutions, so the model will be an approximation to the Friedmann universe. ${ }^{11,32}$

We label the edge lengths in a way appropriate to homogeneous solutions. We distinguish a particular family of spacelike hypersurfaces, where each surface is generated by evolving every vertex in the preceding surface once. The (spacelike) edges lying in these surfaces are called spatial and their lengths are denoted by $l_{i}$. The proper lengths of the (timelike) vertical edges going between these hypersurfaces are denoted by $v_{i}$, and the lengths of the (spacelike) diagonals between these surfaces are denoted by $d_{i}$. In each case, $i$ labels the edges in the class.

Rather than considering the usual description of matter in the Friedmann universe as dust of uniform density, we model it by 120 dust particles each of mass $M / 120$, where $M$ is the total mass, with one particle moving along each of the vertical edges along which the vertices evolve. We note that this entails a kinematical restriction on the particle paths with respect to the geometry, but as the paths made up from the consecutive vertical edges 
will be geodesics in our homogeneous solutions, the particles will indeed move on geodesics. The Regge action is then (in units such that $c=G=1$ )

$$
I=\frac{1}{8 \pi} \sum_{h} A_{h} \epsilon_{h}-\sum_{i} \frac{M}{120} v_{i}
$$

where the second sum is over vertical edges of proper length $v_{i}$. The Regge equations are obtained by varying this with respect to the edge-lengths (cf. Eq. 2.1). Variation with respect to a vertical edge length $v_{i}$ gives

$$
\sum_{h} \frac{\partial A_{h}}{\partial v_{i}} \epsilon_{h}=\frac{\pi M}{15},
$$

and with respect to a diagonal length $d_{i}$ or spatial edge length $l_{i}$ gives

$$
\sum_{h} \frac{\partial A_{h}}{\partial d_{i}} \epsilon_{h}=0, \quad \sum_{h} \frac{\partial A_{h}}{\partial l_{i}} \epsilon_{h}=0 .
$$

We now outline the evolution procedure used here. As discussed in the previous section, the general evolution step involves 13 equations in 13 unknowns, and is expected to allow the approximate freedom to impose four lapse and shift conditions. We will however not consider this general situation, but will instead assume that there exist homogeneous solutions, i.e. that there exist solutions where the lengths $l_{i}$ of the edges in any hypersurface are equal, all $v_{i}$ going between any two adjacent hypersurfaces are equal, and all $d_{i}$ going between any two adjacent hypersurfaces are equal. We will then solve a minimal subset of equations 6.2 and 6.3 needed to generate a solution given this assumption. (In fact we will only use equations of the type of 6.2.) The condition on the $v_{i}$ may be viewed as being merely a choice of lapse, but the conditions on the $d_{i}$ and $l_{i}$ are certainly more than a choice of shift, and involve an assumption about the dynamics of the system. Our approach may be compared with that of Brewin ${ }^{33}$, who made a similar assumption but substituted it into the action in order to derive his equations, thus effectively finding stationary points only with respect to this class of geometries. His and our approaches are correct if and only if the full equations admit such solutions.

To implement this approach we take some hypersurface, consider a vertex [0] in this hypersurface which is connected by spatial edges to the twelve other vertices $[1],[2], \ldots,[12]$, and evolve $[0]$ to the new vertex $\left[0^{*}\right]$. This gives 13 new edges, the vertical one $\left[00^{*}\right]$ and 12 diagonals. The Regge equations available are one for the vertical edge [00*] and 12 for the spatial edges $[01],[02], \ldots,[012]$. (For the first evolution step, the latter 12 equations are not really available since they involve edges in the previous spacetime slice which have not been specified. However, when the process is repeated after the first evolution of all vertices, these equations could be used.) We then assume all spatial edge lengths $l_{i}$ in the surface are equal to some (known) $l_{0}$, we set the proper length of the vertical edge [00*] equal to some $v_{0}$ (i.e. we choose the lapse), and we assume that the twelve diagonals all have the same length, denoted by $d_{0}$. We then use the single Regge equation for the vertical edge $\left[00^{*}\right]$ to solve for $d_{0}$. There are 12 identical triangular hinges, with edge-lengths 
$l_{0}, v_{0}$ and $d_{0}$ and area $A_{010^{*}}$, sharing [00*], and 5 identical 4-simplices meeting on each triangle, so the Regge equation is

$$
v_{0} \cot \theta_{0} \epsilon_{010^{*}}=\frac{\pi M}{90},
$$

where

$$
\cot \theta_{0}=\frac{d_{0}^{2}+l_{0}^{2}-v_{0}^{2}}{4 A_{010^{*}}}
$$

and

$$
\epsilon_{010^{*}}=2 \pi-5 \theta_{010^{*}}
$$

with $\theta_{010^{*}}$ being the hyperdihedral angle at $\left[010^{*}\right]$ in $\left[01230^{*}\right]$ say.

Now consider the evolution of vertex [1] to $\left[1^{*}\right]$. This gives the new vertical edge $\left[11^{*}\right]$, a new spatial edge $\left[0^{*} 1^{*}\right]$, and 11 new diagonals. The Regge equations are one vertical $\left[11^{*}\right]$, one diagonal $\left[10^{*}\right]$, and 11 spatial (the latter 11 not being available the first time around). We again use the lapse freedom to set the length of the vertical edge to be $v_{0}$ (consistent with homogeneity), and we assume that all new diagonals have the length $d_{0}$ (now known). We then solve the Regge equation for the vertical edge [11*] to find the length of the new spatial edge $\left[0^{*} 1^{*}\right]$, denoted by $l_{1}$. In this case there are three types of triangles meeting on $\left[11^{*}\right]$ and two types of 4 -simplex, and the Regge equation reduces to

$$
v_{0}\left[11 \cot \theta_{0} \epsilon_{121^{*}}+\cot \theta_{1} \epsilon_{10^{*} 1^{*}}\right]=\frac{2 \pi M}{15},
$$

where

$$
\begin{gathered}
\cot \theta_{1}=\frac{d_{0}^{2}+l_{1}^{2}-v_{0}^{2}}{4 A_{10^{*} 1^{*}}}, \\
\epsilon_{121^{*}}=2 \pi-2 \theta_{010^{*}}-3 \theta_{121^{*}},
\end{gathered}
$$

and

$$
\epsilon_{10 * 1 *}=2 \pi-5 \theta_{10 * 1 *}
$$

with $A_{10^{*}} 1^{*}$ being the area of triangle $\left[10^{*} 1^{*}\right], \theta_{121^{*}}$ the hyperdihedral angle at $\left[121^{*}\right]$ in $\left[1230^{*} 1^{*}\right]$ and $\theta_{10^{*}} 1^{*}$ the hyperdihedral angle at $\left[10^{*} 1^{*}\right]$ in 4 -simplex $\left[1230^{*} 1^{*}\right]$.

Under our assumption of homogeneity, this completes the evolution from the original hypersurface to the new one, since all vertical edges between the surfaces must have length $v_{0}$, all diagonals have length $d_{0}$, and all spatial edges on the new surface must have length $l_{1}$. The evolution to the next surface may then be carried out in the same fashion, i.e. the next diagonal length $d_{1}$ is found from the vertical equation associated with the evolution of one vertex (given a choice $v_{1}$ of the next vertical edge length), and the next spatial length $l_{2}$ is found from the evolution of an adjacent vertex. Thus the data required to specify a solution of the form we assume are the length $l_{0}$ of the spatial edges on some initial surface, and the vertical length between each pair of consecutive surfaces. In our calculations, the vertical lengths between all consecutive surfaces were chosen to be the same, equal to $v$.

We will discuss the space of solutions in general below, but we commence with those which correspond to the continuum (Friedmann) solution, illustrated by the one shown in 
figure 10. For the purposes of this figure, the spatial edge length $l$ on each hypersurface is converted into an equivalent 3 -sphere radius $a$ by equating the volume of the simplicial hypersurface with that of a smooth 3 -sphere, i.e.

$$
2 \pi^{2} a^{3}=600 \frac{l^{3}}{6 \sqrt{2}},
$$

and this is compared with the scale factor of the Friedmann universe as a function of proper time. (The other variable one might want to plot would be the diagonal length $d$, but in the present case $d$ is virtually identical in magnitude to $l$, due to the small value chosen for the lapse parameter $v$.) Note that the proper time elapsed between two consecutive simplicial hypersurfaces may be defined in different ways. In figure 10 we have used the proper time $\delta t$ as measured along a geodesic running from the centre of a tetrahedron in one surface to the centre of the corresponding tetrahedron in the neighbouring surface, which is given by (see ref. 11, equation 43)

$$
\delta t=\left(v^{2}+\frac{3}{8}\left(l^{\prime}-l\right)^{2}\right)^{1 / 2}
$$

where $l$ and $l^{\prime}$ are the spatial edge lengths on the two surfaces. This gives a slightly slower evolution of the approximate solution as a function of proper time than does the proper time elapsed along a vertical edge, which is just equal to $v$.

The solution shown in figure 10 is a time-symmetric solution of the Regge equations, in which the maximum spatial edge length occurs on two consecutive surfaces, and the other surfaces are symmetrical about these two. That is, if the central surfaces are numbered 0 and 1, then pairs of surfaces numbered $-n$ and $1+n, n$ a positive integer, have the same spatial edge lengths. (The diagonal lengths $d_{i}$ are also symmetrical about the central value of $d_{i}$ going between surfaces 0 and 1.) For this solution it is found that the maximum spatial edge length $l$ satisfies

$$
12 l\left(2 \pi-5 \cos ^{-1}\left(\frac{1}{3}\right)\right)=16 \pi \frac{M}{120} .
$$

This is the Regge analogue of the equation ${ }^{3} R=16 \pi \rho$ (see ref. 32), which of course is the constraint equation applying at the surface of time symmetry in the continuum solution. From 6.11 and 6.13 we find that for the same mass $M$, the equivalent maximum $a$ of the Regge solution is slightly less than the maximum scale factor $a=4 M /(3 \pi)$ of the Friedmann solution. In figure 10 we have compared our Regge solution $(M=10.2)$ with the Friedmann solution $(M=10.0)$ of equal maximum radius $a$.

The Regge equations have a second time-symmetric solution, in which the maximum spatial length $l$ is reached on a single hypersurface, and the other hypersurfaces are symmetric in pairs around this hypersurface. This maximum $l$ is slightly larger than that given by equation 6.13 . There are also solutions which have a maximum $l$ whose value lies between these two, which is reached on one hypersurface, and which are not time-symmetric. 
These are all acceptable models of the Friedmann universe, and on the scale of figure 10 they are indistinguishable from the solution shown there.

The solution in figure 10 has the vertical edge length $v=.0102$. The separate plotted points overlap for most of the solution but may be distinguished toward the ends of the evolution. For $v=.102$, the points in the solution are of course spaced further apart, but they lie on the same curve as a function of proper time. This supports the expectation that $v$ is a gauge degree of freedom.

As was observed previously by Brewin ${ }^{33}$ for his models, the evolution of the Regge universe stops at a finite volume, i.e. solutions cease to exist before the universe collapses to zero volume. Presumably this corresponds to the collapse becoming so fast that the vertical edges would have to become spacelike in order for solutions to exist, but we have not investigated this further. The endpoint is not very sensitive to the value of $v$ used in the calculations.

The Regge equations also have solutions which do not correspond to the Friedmann universe. To begin with, equation 6.7 has two roots for $l_{1}$ for given values of $l_{0}, d_{0}$ and $v_{0}$. One of these roots satisfies the equation

$$
d_{0}^{2}=-v_{0}^{2}+l_{0} l_{1}
$$

which is the same as equation 6.6 of ref. 33. Brewin obtained this equation from the assumption that the 4-simplices in the model are grouped into regular, untwisted 4-prisms. We have not investigated the geometrical interpretation of the other root. The root of equation 6.7 satisfying 6.14 was the one chosen in obtaining the solution shown in figure 10. The other root for $l_{1}$ is larger in the expansion phase and smaller in the contraction phase, giving a solution which evolves about twice as fast as this one.

For given values of $l_{0}$ and $v_{0}$, equation 6.4 typically has four roots for $d_{0}$, which may be naturally grouped into pairs, with one member from each pair giving an expansion of the universe, and the other giving a contraction. One of these pairs of roots leads to the expanding and recontracting solution shown in figure 10, while the other gives a universe which expands without limit from a finite initial volume, plus the corresponding timereversed solution. The physically interesting roots lie in a sharp valley in the centre of a peak of the function on the left hand side of equation 6.4 (considered as a function of $d_{0}$ ), and could not be reliably found with a Newton-Raphson algorithm, so the bisection method was used. Other spurious solutions are allowed in which we jump from one pair of roots to another, or in which the expansion/contraction of the universe reverses arbitrarily. Some of these spurious solutions clearly arise because equation 6.4 is associated with a vertical edge, and so is not sensitive to data prior to the current hypersurface. In particular, an expansion-contraction ambiguity had to be present for this reason. Thus consideration of the equations ignored in our analysis can be expected to eliminate some or all of the spurious solutions.

In summary, we have obtained a solution which is a reasonable approximation to the Friedmann universe, and which compares acceptably to previous solutions ${ }^{11,33}$. We emphasise that our method generalises in a straightforward way to more complicated models, with only a moderate increase in the size of the individual algebraic problems to be solved. The next stage in testing this method is to impose the homogeneity condition on the initial 
data, but only to impose lapse and shift choices on the evolution, and to verify that the full equations do admit the homogeneous solutions discussed here, while excluding the spurious solutions which our truncated treatment permitted. As a first step, one can check how well the solution reported here satisfies the equations which were ignored in obtaining it. Work on this is in progress, together with further investigations of the 5-, 16- and 600-cell universes, as well as work on the evolution of the Kasner universe using a general code for $T^{3}$ topologies based on the "quantity-production lattice." ${ }^{34,17}$ Results will be reported elsewhere.

\section{Conclusions.}

We have described an implicit evolution scheme for Regge calculus. The most important feature of this scheme is the possibility of local evolution for one vertex at a time, a result of the fact that the Regge equation for an edge involves only those simplices containing the edge. Parallel evolution is possible for groups of vertices with no edges in common. Both of these features are very important for numerical calculations.

An important question is whether all the variational equations are independent. In the continuum limit, as we have discussed, one expects the approximate symmetries in the lattice to become exact, giving rise to exact contracted Bianchi identities. The equations will no longer be independent, and the freedom to specify the lapse and shift will be

recovered. Away from the continuum limit, there will be approximate contracted Bianchi identities, giving four conditions per vertex. It will thus probably be best to ignore four equations per vertex and utilize the resulting freedom to specify the discrete lapse and shift, as we did in Sec. 6 with the lapse. As for our results there, it has been shown in other particular $\operatorname{cases}^{29}$ as well, that the evolution is independent of the values of the discrete lapse and shift to high accuracy, an echo of the diffeomorphism symmetry of the continuum.

When we compare the new method with the modification of the prism-based approach described earlier, we see that the symmetries of the lattice are more obvious in the new approach than for the modified prism method, since there is some arbitrariness in dividing the prisms into 4-simplices, and since not all of the symmetries need take the prism-based hypersurfaces into themselves.

The distinction between evolution equations (arising from variation of spacelike edges) and constraint equations (from variation of timelike edges and diagonals) in the prism approach is not present a priori in the new method, although some distinction may arise as a result of consideration of approximate symmetries, or from a choice of a distinguished family of spacelike hypersurfaces. In the prism approach, it is clear which hypersurfaces are meant to correspond to constant times; in the new method, the question of how the hypersurfaces are staggered in time seems to depend on the initial data, which provides the only distinction in the theory between the different classes of hypersurface.

The numerical example described in this paper illustrates the effectiveness of this approach to Regge calculus. Clearly there are many aspects still to be explored; these include the introduction of more general source terms (such as an electromagnetic field, to which our scheme extends essentially unchanged ${ }^{31}$ ), the construction of lattices with different topologies, and the development of efficient ways to present the results. It seems at this 
stage of the development of the theory that it has the attractive features of finite difference methods and also appears to have the advantage of being able to handle the topological complications found in such problems as the two black hole collision and the spatially closed cosmologies. There is a need to test it on evolution problems with many degrees of freedom and to compare its efficiency and accuracy with those of the corresponding finite difference schemes.

\section{Acknowledgements.}

The authors are grateful to I. Pinto and J. C. Miller who organized the very stimulating Workshop on Numerical Applications of Regge Calculus and Related Topics in Amalfi in 1990 where this work was begun. We thank Ian Drummond, Arkady Kheyfets, Pablo Laguna, John Porter and Martin Roček for helpful discussions. WAM acknowledges support from the Air Force Office of Scientific Research. RDS acknowledges support from the National Science Foundation under Grant PHY-9307570. PAT is supported by a fellowship from the Alexander von Humboldt Foundation. RMW thanks Los Alamos National Laboratory for hospitality during the completion of this work.

\section{References.}

[1] K. S. Thorne, "Gravitational radiation," in 300 Years of Gravitation, eds. S. Hawking and W. Israel (Cambridge University Press, 1987) 330-458.

[2] K. S. Thorne, "Sources of Gravitational Waves and Prospects for their Detection," Caltech preprint GRP-234 (1990).

[3] T. Regge "General relativity without coordinates," Nuovo Cimento 19, 558-571 (1961).

[4] J. Cheeger, W. Müller and R. Schrader, "On the curvature of piecewise flat spaces," Comm. Math. Phys. 92, 405-54 (1984).

[5] R. Friedberg and T. D. Lee, "Derivation of Regge's Action from Einstein's Theory of General Relativity," Nucl. Phys. B242, 145-66 (1984).

[6] G. Feinberg, R. Friedberg, T. D. Lee and H. C. Ren, "Lattice Gravity Near the Continuum Limit," Nucl. Phys. B245, 343-68 (1984).

[7] J. W. Barrett, "The Einstein Tensor in Regge's Discrete Gravity Theory," Class. Quantum Grav. 3 203-6 (1986).

[8] J. W. Barrett, "A convergence Result for Linearised Regge Calculus," Class. Quantum Grav 5, 1187-92 (1988); and J. W. Barrett and R. M. Williams, "The convergence of Lattice Solutions of Linearised Regge Calculus," Class. Quantum Grav. 5, 1543-56 (1988).

[9] L. Brewin, "Equivalence of the Regge and Einstein Equations for Almost Flat Simplicial Spacetimes," Gen. Rel. Grav. 21, 565-83 (1989).

[10] R. M. Williams and P. A. Tuckey, "Regge calculus: a brief review and bibliography," Class. Quantum Grav. 9, 1409-22 (1992).

[11] P. A. Collins and R. M. Williams, "Dynamics of the Friedmann Universe Using Regge Calculus," Phys. Rev. D7, 965-71 (1973). 
[12] L. Brewin, The Regge Calculus in Numerical Relativity, Ph. D. Thesis Monash Univ. (1983).

[13] J. D. Porter, "A New Approach to the Regge Calculus: I. Formalism," Class. Quantum Grav. 4, 375-89 (1987).

[14] J. D. Porter, "A New Approach to the Regge Calculus: II. Application to SphericallySymmetric Vacuum Space-Times," Class. Quantum Grav. 4, 391-410 (1987).

[15] M. R. Dubal, Numerical Computations in General Relativity, Ph. D. Thesis SISSA Trieste (1987).

[16] W. A. Miller and J. A. Wheeler, "4-Geodesy," Nuovo Cimento 8, 418-34 (1985).

[17] W. A. Miller, "Geometric Computation: Null-Strut Geometrodynamics and the Inchworm Algorithm," in Dynamical Spacetimes and Numerical Relativity, ed. J. Centrella (Cambridge University Press, 1986) 256-303.

[18] A. Kheyfets, W. A. Miller and J. A. Wheeler, "Null-Strut Calculus: The First Test," Phys. Rev. Lett., 61, 2042-45 (1988).

[19] A. Kheyfets, N. J. LaFave and W. A. Miller, "Null-Strut Calculus I: Kinematics," Phys. Rev. D41, 3628-36 (1990).

[20] A. Kheyfets, N. J. LaFave and W. A. Miller, "Null-Strut Calculus II: Dynamics," Phys. Rev. D41, 3637-51 (1990).

[21] W. A. Miller developed a (3+1)-dimensional initial-value code for null-strut calculus (Oppie-3) and applied it to the Kasner cosmology, Unpublished (1990).

[22] R. D. Sorkin, "Time-Evolution Problem in Regge Calculus," Phys. Rev. D12, 385-96 (1975).

[23] P. A. Tuckey, "The Construction of Sorkin Triangulations," Class. Quantum Grav. 10, L109-13 (1993).

[24] J. W. Barrett, "A Mathematical Approach to Numerical Relativity," in Approaches to Numerical Relativity, Proc. of the International Workshop on Numerical Relativity Southampton December 1991, ed. R. d'Inverno (Cambridge: Cambridge University Press, 1992) 103-13.

[25] W. A. Miller, "The Geometrodynamic Content of the Regge Equations as Illuminated by the Boundary of a Boundary Principle," Found. Phys. 16, 143-69 (1986).

[26] C. Misner, K. S. Thorne and J. A. Wheeler, Gravitation (W. H. Freeman and Co., 1973) 505-8.

[27] H. Kurki-Suonio, P. Laguna and R. A. Matzner, "Inhomogeneous Inflation: Numerical Evolution," Phys. Rev. D48, 3611-24 (1993).

[28] M. R. Dubal, "Relativistic Collapse Using Regge Calculus: I. Spherical Collapse Equations," Class. Quantum Grav. 6, 1925-41 (1989).

[29] M. Galassi, "Lapse and Shift in Regge Calculus," Phys. Rev. D47, 3254-64 (1993).

[30] R. D. Sorkin, Development of Simplicial Methods for the Metrical and Electromagnetic Fields, Ph.D. Thesis California Institute of Technology (1974) (available from University Microfilms, Ann Arbor, Michigan). 
[31] R. D. Sorkin, "The Electromagnetic Field on a Simplicial Net," J. Math. Phys. 16, 2432-40 (1975); and Erratum, J. Math. Phys. 19, 1800 (1978).

[32] J. A. Wheeler, "Geometrodynamics and the Issue of the Final State," in Relativity, Groups and Topology, eds. B. DeWitt and C. DeWitt (New York: Gordon and Breach, 1964) 463-500.

[33] L. Brewin, "Friedmann Cosmologies via the Regge Calculus," Class. Quantum Grav. 4, 899-928 (1987).

[34] M. Galassi, Private Communication (1994).

[35] J. B. Hartle, "Simplicial Minisuperspace I. General Discussion," J. Math. Phys. 26, 804-18 (1985); "Simplicial Minisuperspace II. Some Classical Solutions on Simple Triangulations," J. Math. Phys. 27, 287-95 (1986).

[36] R. D. Sorkin, "A Finitary Substitute for Continuous Topology?", Int. J. Th. Phys. 30, 923-47 (1991)

[37] S. M. Lewis, Regge Calculus: Applications to Classical and Quantum Gravity, Ph.D. Thesis Univ. of Maryland at College Park (1982).

[38] J. W. Barrett and P. E. Parker, "Smooth Limits of Piecewise-Linear Approximations," J. Approx. Theory 76, 107-22 (1994).

[39] M. Roček and R. M. Williams, "Quantum Regge Calculus," Phys. Lett. 104B, 31-7 (1981); "Introduction to Quantum Regge Calculus," in Quantum Structure of Space and Time, eds. M. J. Duff and C. J. Isham (Cambridge: Cambridge University Press, 1982); "The Quantization of Regge Calculus," Z. Phys. C 21, 371-81 (1984).

[40] T. Piran and A. Strominger, "Solutions of the Regge Equations," Class. Quantum Grav. 3, 97-102 (1986)

[41] C. P. Rourke and B. J. Sanderson, Introduction to Piecewise-Linear Topology (Springer, 1982), proposition 2.9 


\section{Figure Captions}

Fig. 1 Part of a 2-dimensional simplicial spacelike surface surrounding a vertex.

Fig. 2 A (2+1)-dimensional "tent" erected over the vertex shown in Fig. 1. The tent consists of six tetrahedra sharing a common "vertical" edge (darkened dashed edge).

Fig. 3 A section of a (3+1)-dimensional "tent" erected over a vertex in a spacelike hypersurface. For each tetrahedron in the hypersurface, a 4-simplex is constructed with apex at the new vertex. Due to the complexity of the diagram we show here only one of the numerous simplices sharing the common "vertical" edge.

Fig. 4 Evolution of a surface in $1+1$ dimensions, by advancing the vertices one-by-one.

Fig. 5 Evolution of a surface in 1+1 dimensions, by advancing the vertices in parallel (cf. Fig. 4).

Fig. 6 To ensure an accurate evolution step (in accordance with the Courant limit) we must choose the lapse and shift conditions so that the "tent" lies within the "light cone on" its spacelike base as illustrated here in its $(1+1)$-dimensional form. (Notwithstanding the implicit character of our scheme, we believe it will still be subject to a Courant limit as a result of its strict locality.)

Fig. 7 Sorkin's lattice for $S^{1} \times R$.

Fig. 8 The 16 -cell tessellation $\beta_{4}$ of $S^{3}$.

Fig. 9 A prism used in the evolution of $\beta_{4}$. It consists of two spacelike tetrahedra with corresponding vertices joined by timelike edges.

Fig. 10 Comparison of the effective radius $a$ (as defined in the text, see equation 6.11) of the 600-cell model (lower, dotted line) with the scale factor of the Friedmann universe (upper, solid line) as functions of proper time. The Regge model has $v=.0102$ and $M=10.2$, and $a$ is plotted versus proper time elapsed at the centre of a tetrahedron (see text, equation 6.12); the comparison Friedmann universe has $M=10$. (Units are such that $c=G=1$.) 
FIGURE 1

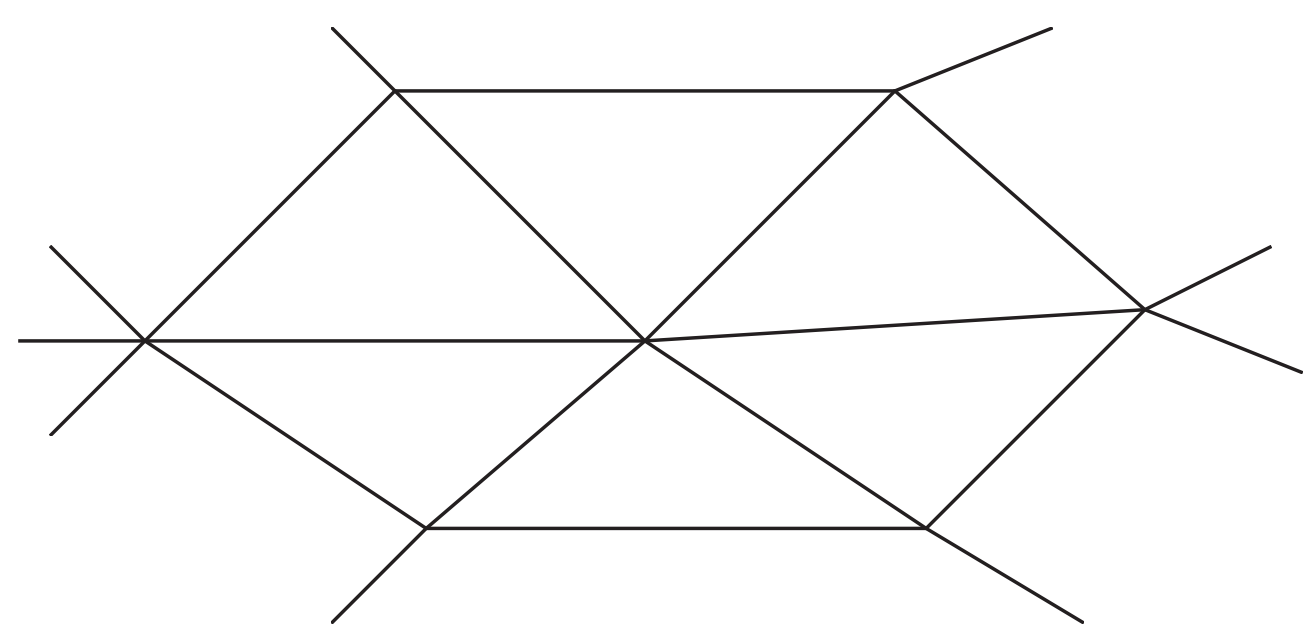




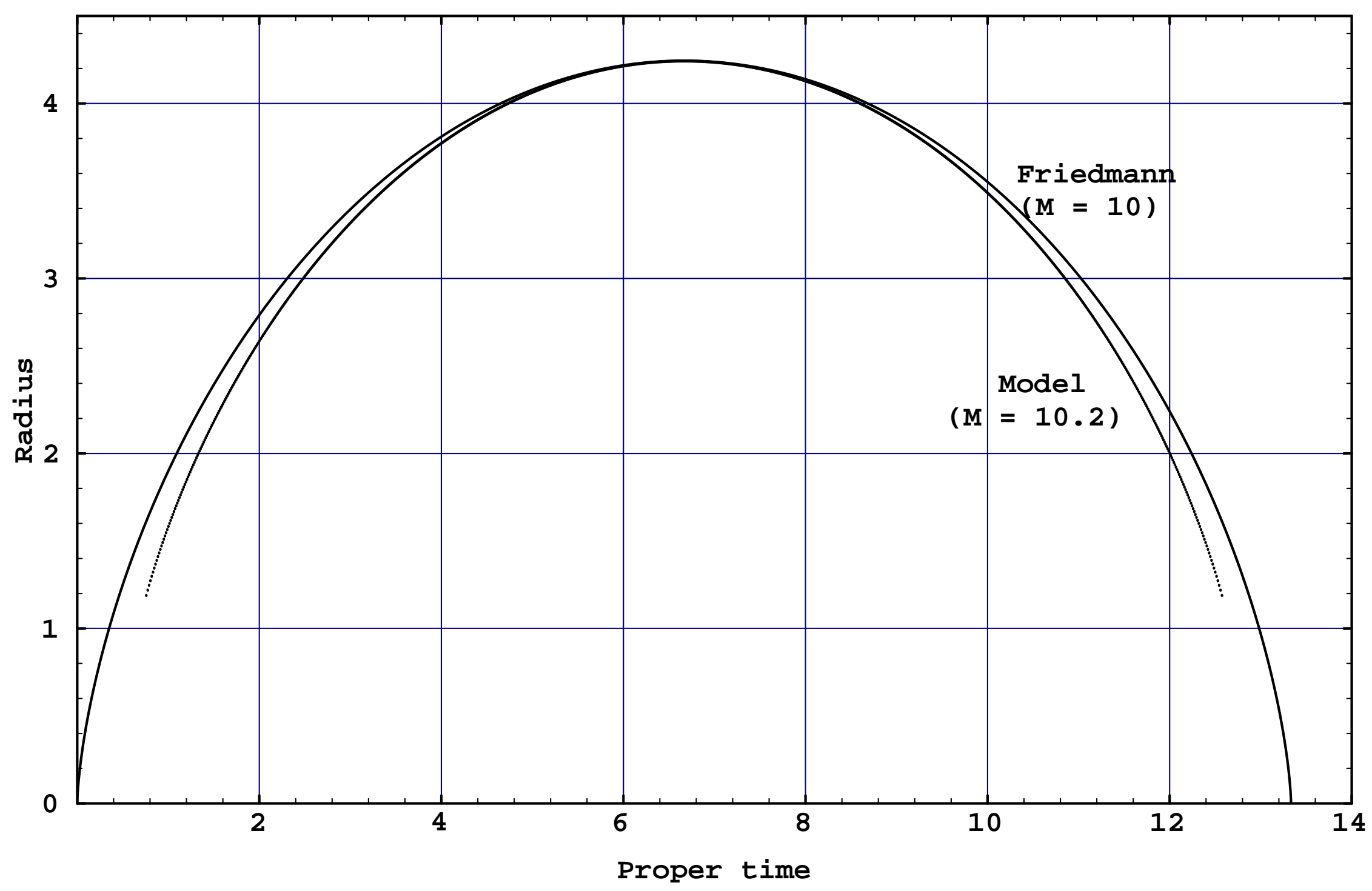

四 


\section{FIGURE 2}

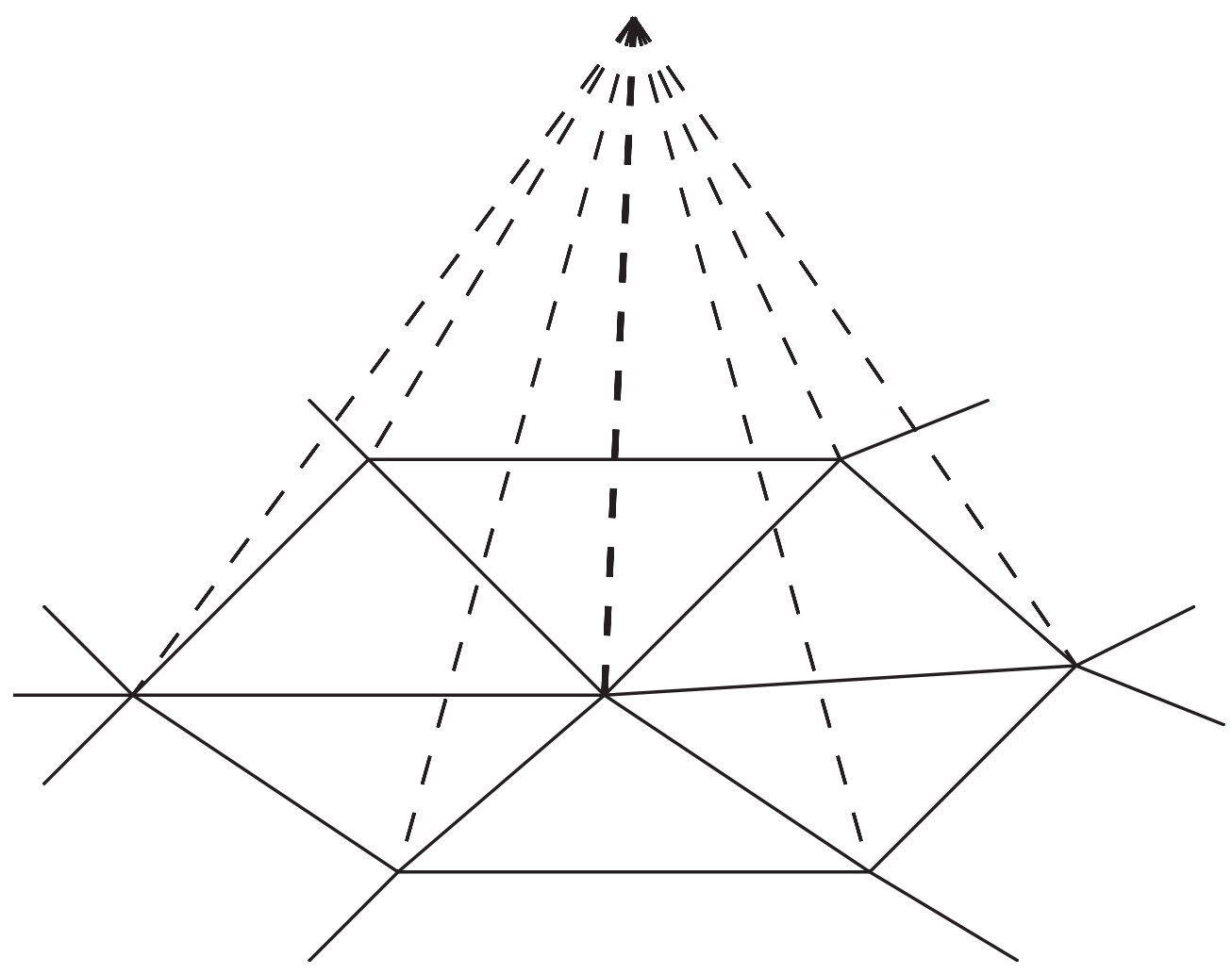




\section{FIGURE 3}

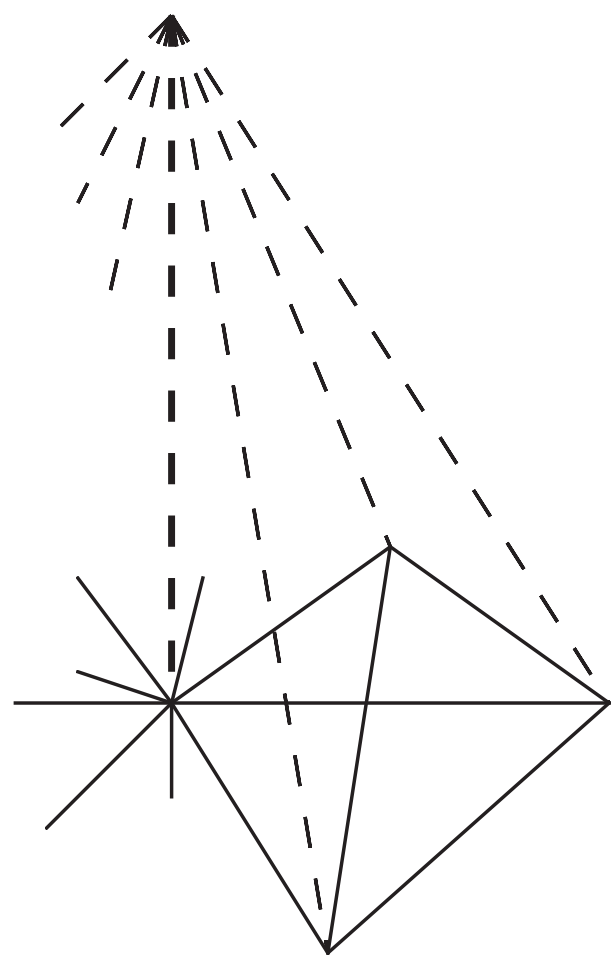


FIGURE 4

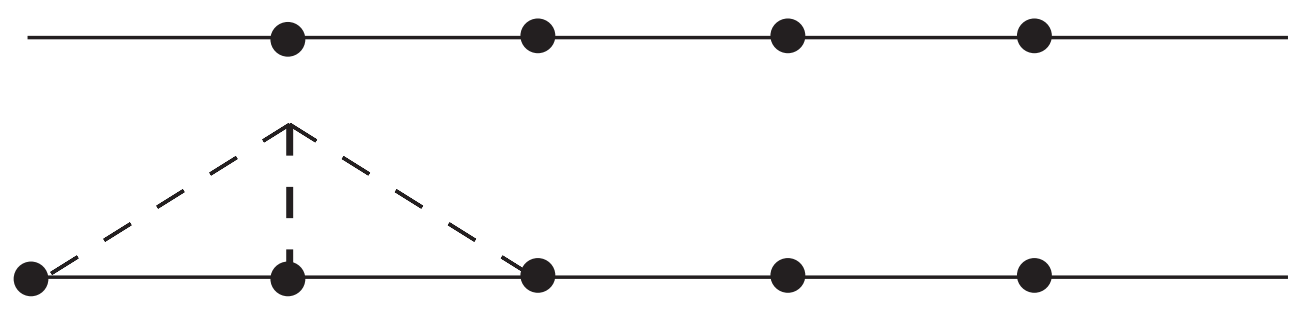

Step 1
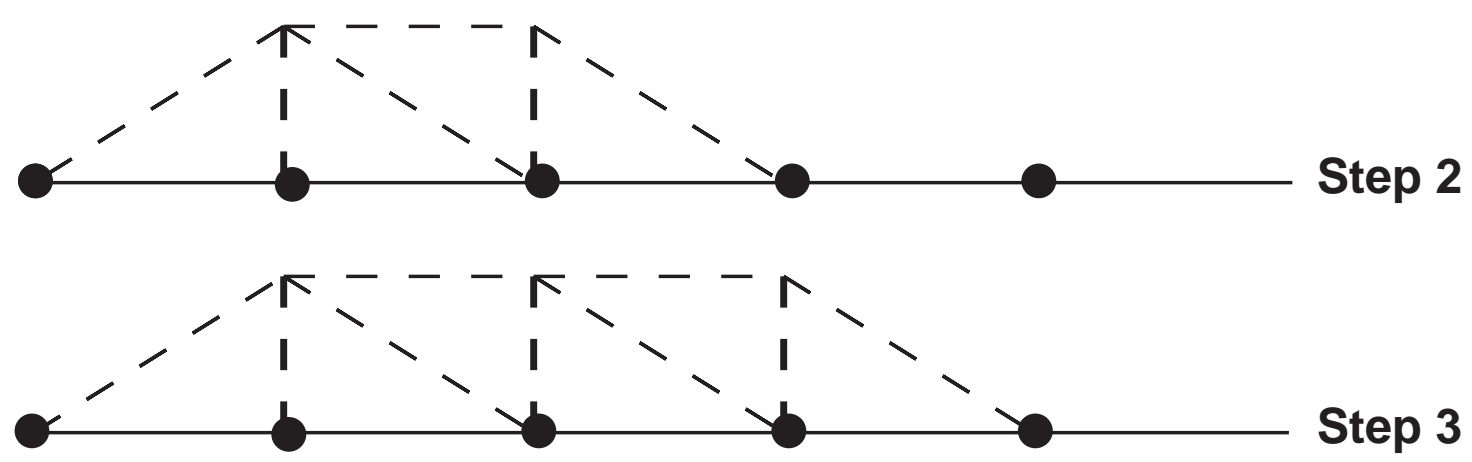

and so on 


\section{FIGURE 5}

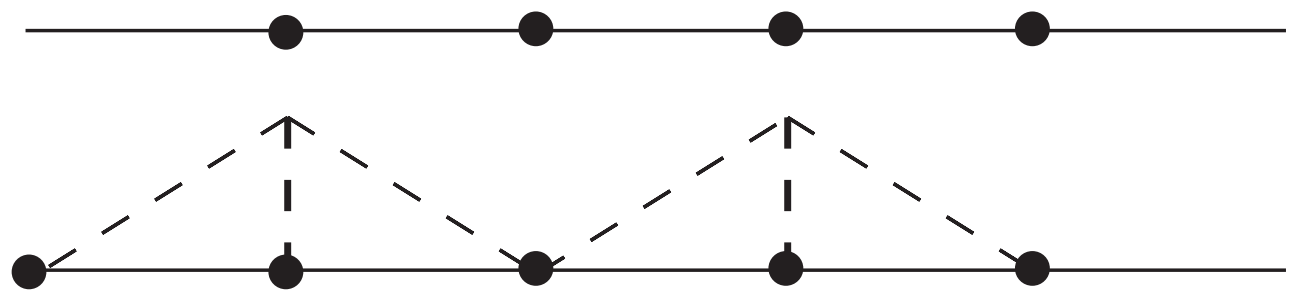

Step 1

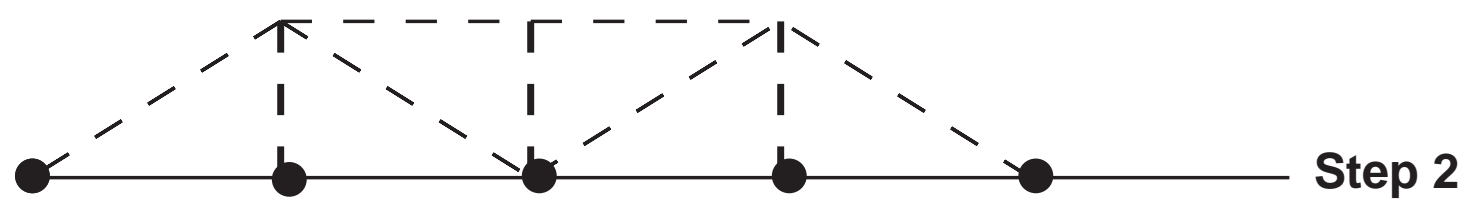

and so on 


\section{FIGURE 6}

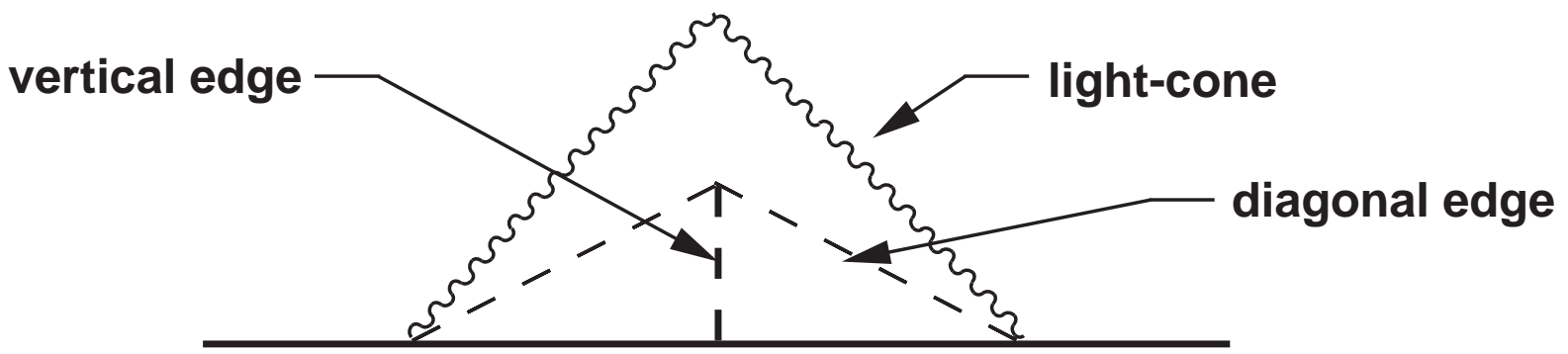


FIGURE 7

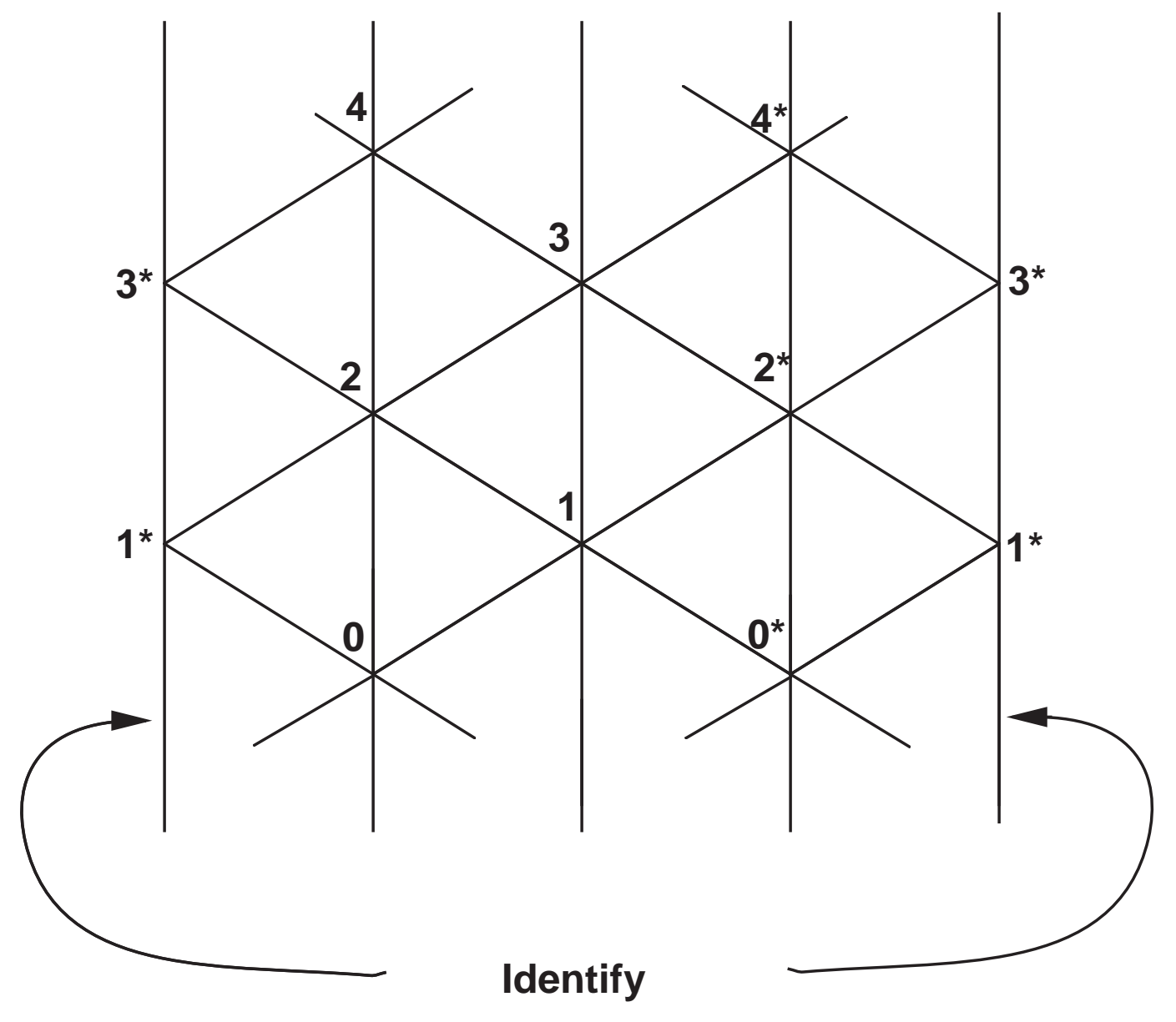


FIGURE 8

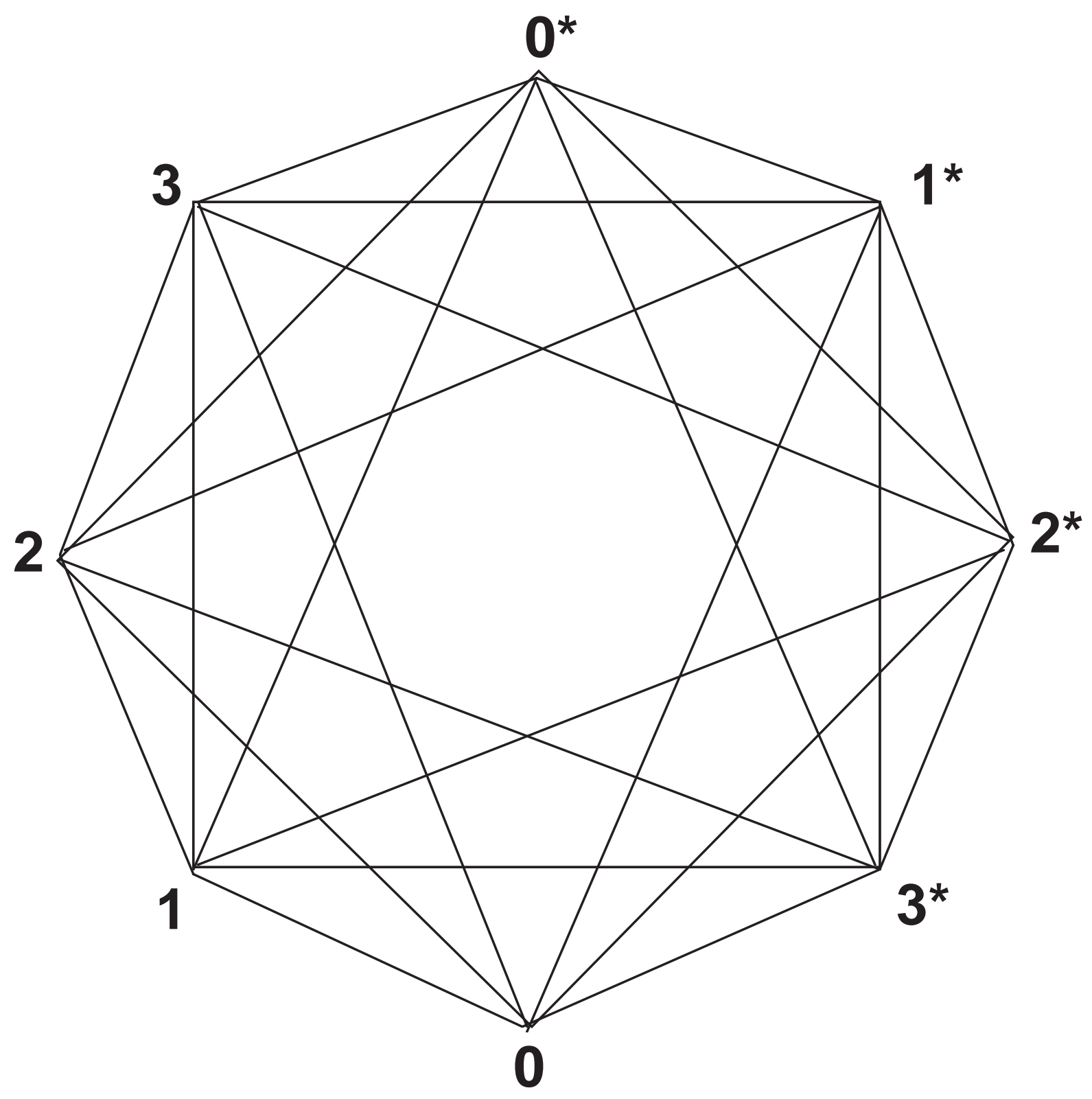


FIGURE 9

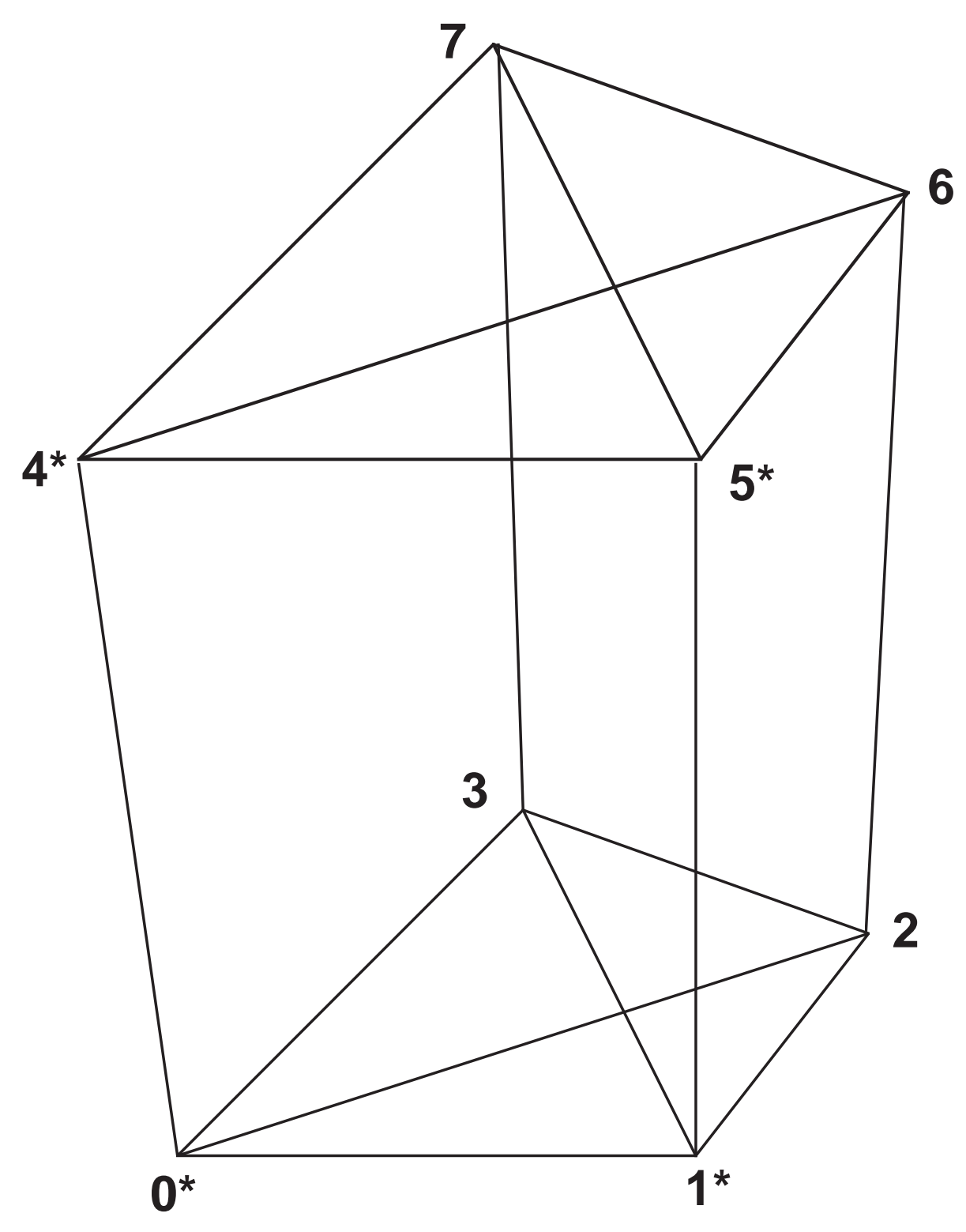


This figure "fig1-1.png" is available in "png" format from: http://arxiv.org/ps/gr-qc/9411008v1 
This figure "fig2-1.png" is available in "png" format from: http://arxiv.org/ps/gr-qc/9411008v1 
This figure "fig1-2.png" is available in "png" format from: http://arxiv.org/ps/gr-qc/9411008v1 
This figure "fig2-2.png" is available in "png" format from: http://arxiv.org/ps/gr-qc/9411008v1 
This figure "fig1-3.png" is available in "png" format from: http://arxiv.org/ps/gr-qc/9411008v1 
This figure "fig2-3.png" is available in "png" format from: http://arxiv.org/ps/gr-qc/9411008v1 
This figure "fig1-4.png" is available in "png" format from: http://arxiv.org/ps/gr-qc/9411008v1 
This figure "fig2-4.png" is available in "png" format from: http://arxiv.org/ps/gr-qc/9411008v1 
This figure "fig1-5.png" is available in "png" format from: http://arxiv.org/ps/gr-qc/9411008v1 
This figure "fig2-5.png" is available in "png" format from: http://arxiv.org/ps/gr-qc/9411008v1 